\title{
Risk Stratification Models to Predict Hypertensive Disorders of Pregnancy: Additive Value of Standard Electrocardiography
}

\author{
Monica Trapasso ${ }^{1}$, Enrica Angeli ${ }^{2}$, Paolo Verdecchia ${ }^{3}$, Gianpaolo Reboldi ${ }^{1}$ and Fabio Angeli ${ }^{*}$ \\ ${ }^{1}$ Department of Medicine, University of Perugia, Perugia, Italy \\ ${ }^{2}$ Department of Obstetrics and Gynecology, Hospital of Foligno, Foligno, Italy \\ ${ }^{3}$ Department of Internal Medicine, Hospital of Assisi, Assisi, Italy \\ ${ }^{4}$ Department of Cardiology and Cardiovascular Pathophysiology, University and S.M. della Misericordia Hospital of \\ Perugia, Perugia, Italy
}

*Corresponding author: Fabio Angeli, MD, Department of Cardiology and Cardiovascular Pathophysiology, University and S.M. della Misericordia Hospital of Perugia, 06100 Perugia, Italy, Tel: +39-075-5782394, E-mail: angeli.internet@gmail.com

\begin{abstract}
Hypertension disorders complicate up to $10 \%-11 \%$ of all pregnancies and remain leading causes of poor outcome, including placental abruption, organ failure, cerebrovascular accident and disseminated intravascular coagulation. These disorders are also associated with increased risk of perinatal death, fetal intrauterine growth restriction, and prematurity/preterm delivery.

Epidemiological evidences supporting the worse prognosis associated with hypertension in pregnancy provide a strong basis for developing perinatal morbidity and mortality risk prediction models.

Of the many risk markers for hypertensive disorders, some are known at booking and increase the risk of hypertensive disorders two- to four-fold. They include pre-existing hypertension, diabetes mellitus and renal disease, previous preeclampsia, antiphospholipid antibody syndrome, overweight/obesity, inter-pregnancy interval $\geq 10$ years, and multiple pregnancies.

Recently, the additive value of some instrumental techniques (including uterine artery Doppler velocimetry, electrocardiography and ambulatory blood pressure monitoring) and their combinations with maternal factors and biochemical markers to refine risk stratification for hypertensive disorders in pregnancy has also been evaluated.

The main aim of our systematic review was to summarize the present state of knowledge in this active area of broad interest. Specifically, we aimed to provide an overview of recent contributions on the role of electrocardiography for the identification of women at increased risk of hypertensive complications during pregnancy.
\end{abstract}

Briefly, current Guidelines recommend performing a 12lead electrocardiogram in order to evaluate the presence of left ventricular hypertrophy in pregnant women. Nevertheless, some abnormal electrocardiographic patterns, particularly in the first trimester of pregnancy, may increase the risk of maternal and neonatal complications. In this context, left atrial abnormalities in lead $V_{1}$ have been suggested as independent predictors of hypertensive disorders and other pregnancy complications including fetal growth restriction, HELLP (hemolysis, elevated liver enzymes, low platelets) syndrome, placental abruption, stillbirth, premature delivery and neonatal death.

Available data support the notion that risk stratification for hypertensive disorders might be improved in the first-trimester of pregnancy using standard electrocardiography in combination with maternal characteristics and history. An effective screening for hypertensive disorders is useful to identify women that would potentially benefit from a closer surveillance and from prophylactic pharmacological interventions.

\section{Keywords}

Electrocardiography, Pregnancy, Hypertension, Eclampsia, Pre-eclampsia, Hypertensive disorders, Prognosis

Abbreviations
ABPM: Ambulatory Blood Pressure Monitoring; BP: Blood
Pressure; Cl: Confidence Interval; ECG: Electrocardiog-
raphy; HELLP: Hemolysis, Elevated Liver Enzymes, Low
Platelets; HR: Heart Rate; MAP: Mean Arterial Pressure;
OR: Odds Ratio; RAS: Rennin Angiotensin System; SOGC:
Society of Obstetricians and Gynecologists of Canada

Citation: Trapasso M, Angeli E, Verdecchia P, Reboldi G, Angeli F (2017) Risk Stratification Models to Predict Hypertensive Disorders of Pregnancy: Additive Value of Standard Electrocardiography. Int J Womens Health Wellness 3:061. doi.org/10.23937/2474-1353/1510061

Received: September 14, 2017: Accepted: October 11, 2017: Published: October 13, 2017

Copyright: (c) 2017 Trapasso M, et al. This is an open-access article distributed under the terms of the Creative Commons Attribution License, which permits unrestricted use, distribution, and reproduction in any medium, provided the original author and source are credited. 


\section{Introduction}

As recommended by the National High Blood Pressure (BP) Education Program Working Group on High BP in Pregnancy [1], hypertensive disorders during pregnancy are classified into 4 categories:

- Chronic hypertension

- Preeclampsia-eclampsia

- Preeclampsia superimposed on chronic hypertension

- Gestational hypertension (transient hypertension of pregnancy or chronic hypertension identified in the latter half of pregnancy).

In 2014, the Society of Obstetricians and Gynecologists of Canada (SOGC) [2] released revised guidelines that simplified the classification of hypertension in pregnancy into the following categories: (i) Preexisting hypertension; (ii) Gestational hypertension; (iii) Preeclampsia; and (iv) "Other hypertensive effects" on the basis of different diagnostic considerations.

Determining the true incidence of the hypertensive disorders of pregnancy is complicated by variations in the reported classification of the disorders. The most commonly cited and accepted estimate of hypertensive disorder of pregnancy occurrence is around $10-11 \%$ [3].

Hypertensive disorders of pregnancy can develop during pregnancy or delivery and are major causes of maternal and perinatal morbidity and mortality [4-6]. Specifically, hypertensive disorders can trigger some severe forms of maternal complications, such as cardiovascular and cerebrovascular diseases, liver and kidney failure, placental abruption, disseminated intravascular coagulation and HELLP (Hemolysis, Elevated Liver Enzymes, Low Platelets) syndrome. Under these circumstances, the placenta dysfunction may occur, leading to fetal growth restriction, fetal distress, preterm birth, intrauterine fetal demise, stillbirth and neonatal asphyxia [1]. Moreover, hypertensive disorders of pregnancy are associated with increased risk of future chronic hypertension [4-7].

Over the past few decades, several studies have been conducted to identify the pregnant women at higher risk of hypertensive disorders.

This review summarizes the present state of knowledge in this active area of broad interest. Specifically, we aimed to provide an overview of recent contributions on the role of instrumental techniques for the identification of women at increased risk of hypertensive complications during pregnancy.

To this purpose, we searched for clinical studies and systematic overviews using research methodology filters $[8,9]$. The following research terms were used: "hypertension", "pregnancy", "gestational hypertension", "eclampsia", "pre-eclampsia", "blood pressure" and prognosis". We also checked the reference list of identified articles and previous systematic reviews to find other relevant studies.

\section{Blood Pressure Measurements}

Traditionally, diagnosis and management of arterial hypertension are based on BP measurements taken in the physician's office. Women should be allowed to sit quietly for 5-10 minutes before each BP measurement $[1,2]$. Of note, compression on the inferior vena cava by the gravid uterus while the patient is supine can alter readings substantially, leading to an underestimation of the $\mathrm{BP}$.

Similarly, BP measured in the left lateral position may yield falsely low values if the BP is measured in the higher arm, unless the cuff is carefully maintained at the level of the heart. Thus, BP should be measured in the sitting position, with the cuff at the level of the heart. Korotkoff sounds I (the first sound) and V (the disappearance of sound) should be used to denote the systolic BP and diastolic BP, respectively. Maternal systolic $\mathrm{BP}$ greater than $160 \mathrm{mmHg}$ or diastolic BP greater than $110 \mathrm{mmHg}$ denotes severe disease $[1,2]$.

Home BP measurements are recommended in the pregnant population. In this context, some automated BP devices have been validated in pregnancy (http:// www.dableducational.org/sphygmomanometers/devices_1_clinical.html\#ClinTable). We strongly recommend using validated devices for assessment of hypertension severity and control during pregnancy.

A large clinical study involving 9149 women with singleton pregnancies examined the performance of screening for hypertensive disorders in pregnancy comparing systolic BP, diastolic BP, and Mean Arterial Pressure (MAP) measured by validated automated devices. Specifically, the best performance in screening was provided by MAP [10]. The detection rate of early-preeclampsia at a $10 \%$ false-positive rate increased from $47 \%$ in screening by maternal factor-derived a priori risk alone to $76 \%$ in screening by its combination with MAP. The respective detection rates for late-pre-eclampsia increased from 41 to $52 \%$ and for gestational hypertension increased from 31 to $48 \%$ [10].

\section{Risk Quantification: A Combined Approach}

Epidemiological evidence supporting the worse prognosis associated with hypertension in pregnancy provides a strong basis for developing risk prediction models to identify women at increased risk for hypertensive disorders. These women may require a closer surveillance and preventive treatments [11].

To date, several risk factors for development of hypertensive disorders in pregnancy have been described, including preexisting chronic hypertension, chronic vascular and renal disease, connective tissue disease, dyslipidemia, diabetes, obesity, age more than 40 years, multifetal gestation, family history of preeclampsia and fetal growth restriction [7,12-20].

To improve the sensitivity and the positive predictive 


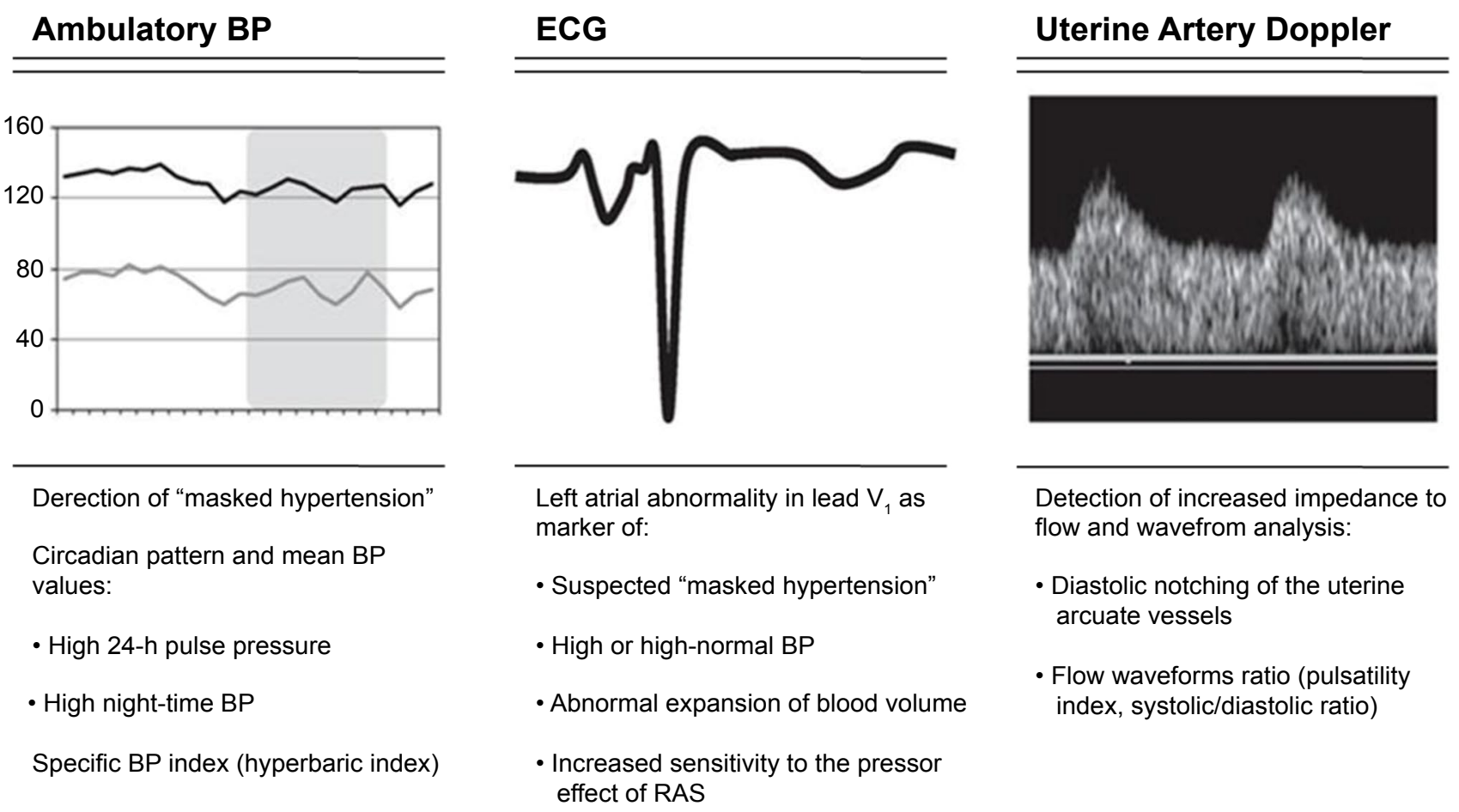

Figure 1: Instrumental techniques used to identify women at increased risk of hypertensive disorders during pregnancy. For each test, main parameters tested as predictors of hypertensive disorders are reported [7].

values of screening programs, some biochemical markers (including laboratory markers, urinary proteomics, markers of inflammation, antiphospholipid antibodies, and coagulation factors) have been investigated as potential predictors of hypertensive disorders during pregnancy [7,21-24].

Particularly, an experimental study including 120 normotensive pregnant and 60 pregnancy-induced hypertensive women reported that pregnancy-induced hypertension was associated with significantly lower levels of serum total calcium, urinary calcium and magnesium excretions and plasma renin activity [25].

To further clarify these findings, a systematic overview and meta-analysis investigated the relationship between serum zinc, magnesium, and calcium levels and pregnancy-induced hypertension [26].

Briefly, a total of 14 studies were included and results indicated that patients with pregnancy-induced hypertension had lower serum zinc, calcium, and magnesium concentration than healthy gravidas [26].

Such results have some implications on the aetiology of hypertension in pregnancy and suggest that mineral supplementation during the antenatal period may influence prevention and treatment of hypertensive disorders of pregnancy.

Although several circulating and urinary markers have been proposed as potential predictors of hypertensive disorders during pregnancy, any single test may lack predictive value or practical utility to be applied at large [7].
Moreover, early prediction of hypertensive disorders in healthy and initially normotensive pregnant women remains problematic, partly because severe forms such as pre-eclampsia and eclampsia are etiologically complex and heterogeneous conditions $[7,11]$.

In this context, evidence suggests that an effective screening for the development of hypertensive disorders can be provided in the first-trimester of pregnancy. Instrumental/imaging techniques might improve the accuracy of multivariable predictive models for the prediction of hypertensive disorders of pregnancy, especially for the more severe forms. Particularly, screening by a combination of maternal risk factors, uterine artery Doppler, out-of-office BP measurements and standard Electrocardiography (ECG) can identify women at increasing risk for the development of hypertensive complications (Figure 1) [7].

\section{ABPM}

Ambulatory BP Monitoring (ABPM) has become a clinically useful modality in BP assessment in pregnancy; efforts have been made to predict hypertensive disorders of pregnancy by using ABPM [7].

In a prospective cohort study by Bellomo, et al. 254 women without preexisting hypertension and not treated with antihypertensive drugs aid with high $(n=148)$ or normal ( $n=106$ ) office BP underwent 24-hour noninvasive ABPM [27]. The Authors showed that in women with elevated BP during their third trimester of pregnancy, 24-hour BP was superior to office BP to predict the outcome. The sensitivity and specificity of 24-hour 
BP were $87.5 \%$ and $77.7 \%$; for office BP measurement, 91.6\% and 55.4\%; for 24-hour proteinuria, $47.2 \%$ and $100 \%$, respectively, for the prediction of pre-eclampsia [27].

More recently, the usefulness of ABPM during pregnancy was confirmed by Brown, et al. Specifically, 122 pregnant women who were considered at high risk for the development of pre-eclampsia underwent 24-hour ABPM between 18 and 30 weeks gestation, while their condition was normotensive according to routine mercury sphygmomanometry [28]. One hundred sixty-four healthy primigravid women who were considered at usual risk for preeclampsia underwent the same tests as a parallel study [28]. Routine BP, awake and sleep average BP, and 24-hour mean average BP were entered into multiple logistic regression as predictors of either preeclampsia or gestational hypertension; significant variables were then tested by a series of receiver operator curves. Results showed that awake and sleeping BP was higher in mid-pregnancy in women who later developed preeclampsia or gestational hypertension [28]. In particular, hypertension during sleep was a common finding in women with hypertensive disorders of pregnancy. These women also showed higher awake BP and a greater frequency of adverse maternal and fetal outcomes [29].

Furthermore, Hermida, et al. [30] have shown that, in pregnancy, the hyperbaric index (area of BP excess above the upper limit of a time-specified tolerance interval) derived from ABPM was superior to office measurements for predicting the outcome of pregnancy.

\section{Uterine Artery Doppler}

Normal placentation is achieved through successful trophoblast invasion of the maternal decidua and myometrium via the dilated spiral arteries. In the process, a low resistance vascular bed with a high blood flow is created. Physiological changes during pregnancy convert the spiral arteries from small muscular arteries to dilated uteroplacental vessels, which are able to accommodate the hemodynamic forces of pregnancy. Unsuccessful trophoblast invasion, with consequent under perfusion of the placenta, leads to the release of hormones into the maternal circulation which is believed to be the underlying mechanism for the development of hypertensive disorders [7,31-33].

The use of Doppler imaging permits non-invasive evaluation of the uteroplacental circulation by comparing systolic and diastolic waveforms. In this context, the assessment of uteroplacental circulation by Doppler ultrasonography of the uterine arteries has been reported in numerous studies as a promising technique for predicting the level of risk for hypertensive disorders in pregnancy [34-37].

A systematic review and meta-analysis [37] of studies in which Doppler assessment of the uterine arteries was used, showed that abnormal uterine artery waveform was a strong predictor of preeclampsia. In particular, an increased pulsatility index with notching was an independent predictor of pre-eclampsia with a positive likelihood ratio equal to 21.0 among high-risk patients and 7.5 among low-risk patients. It was also a predictor of overall (positive likelihood ratio 9.1) and severe (positive likelihood ratio 14.6) intrauterine growth restriction among low-risk patients [37].

\section{ECG}

Pregnancy may induce some ECG changes which may regress later in pregnancy or following delivery [7,38-48]. Main ECG changes induced by pregnancy are reported in Figure 2.

Some studies have investigated the ECG changes in pregnant women with hypertensive disorders during pregnancy $[39,47-51]$. To date, there is evidence that hypertensive disorders of pregnancy can be predicted by changes in P-wave morphology and QT interval $[39,48]$.

QT interval seems to be unaffected by normal pregnancy [38]. Conversely, pregnancies with abnormal uterine perfusion that developed pathological outcomes seem to be paralleled by changes in ventricular repolarization that may precede clinical symptoms [50].

In this context, a prospective study by Isezuo and Ekele [49] including 60 pregnant Nigerian women (mean age $19.5 \pm 4.2$ years) showed that eclamptic patients had higher frequency of abnormal QTc (46.7\% versus $6.6 \%$, odds ratio [OR]: 9.2; 95\% Confidence Interval [CI]: $1.61-68.48, p=0.01$ ) as measured on the surface ECG when compared to women with normal pregnancy [49].

Similarly, Raffaelli and co-workers [51] evaluated the effect of pre-eclampsia on electrical cardiac activity on Caucasian women from Italy. They demonstrated that pregnancies complicated by pre-eclampsia had a significant alteration of ventricular repolarization. They compared pre-partum ECGs of 76 consecutive pre-eclamptic women with those of 76 healthy pregnant women. All of the routine ECG parameters were considered, and ventricular repolarization was assessed by QT interval and QT dispersion (QTd).

Pre-eclamptic women showed a lower heart rate (HR, $77.4 \pm 14.3$ vs. $81.6 \pm 11.0$ beats per minute [bpm]), a longer mean QTc interval $(442.7 \pm 26.7$ vs. $423.7 \pm 20.7$ msec) and a higher QTd (24.0 vs. $22.0 \mathrm{msec}$ ) than the control group [51]. Moreover, P-wave duration was significantly longer in the pre-eclamptic women than in the control group of normal pregnancy [51].

Just recently, a case-control study from Kirbas and co-workers [52] evaluated $P$ wave parameters to determine the association between preeclampsia and future cardiovascular risk and to study the possible correlation between $\mathrm{P}$ waves and severity of preeclampsia among 


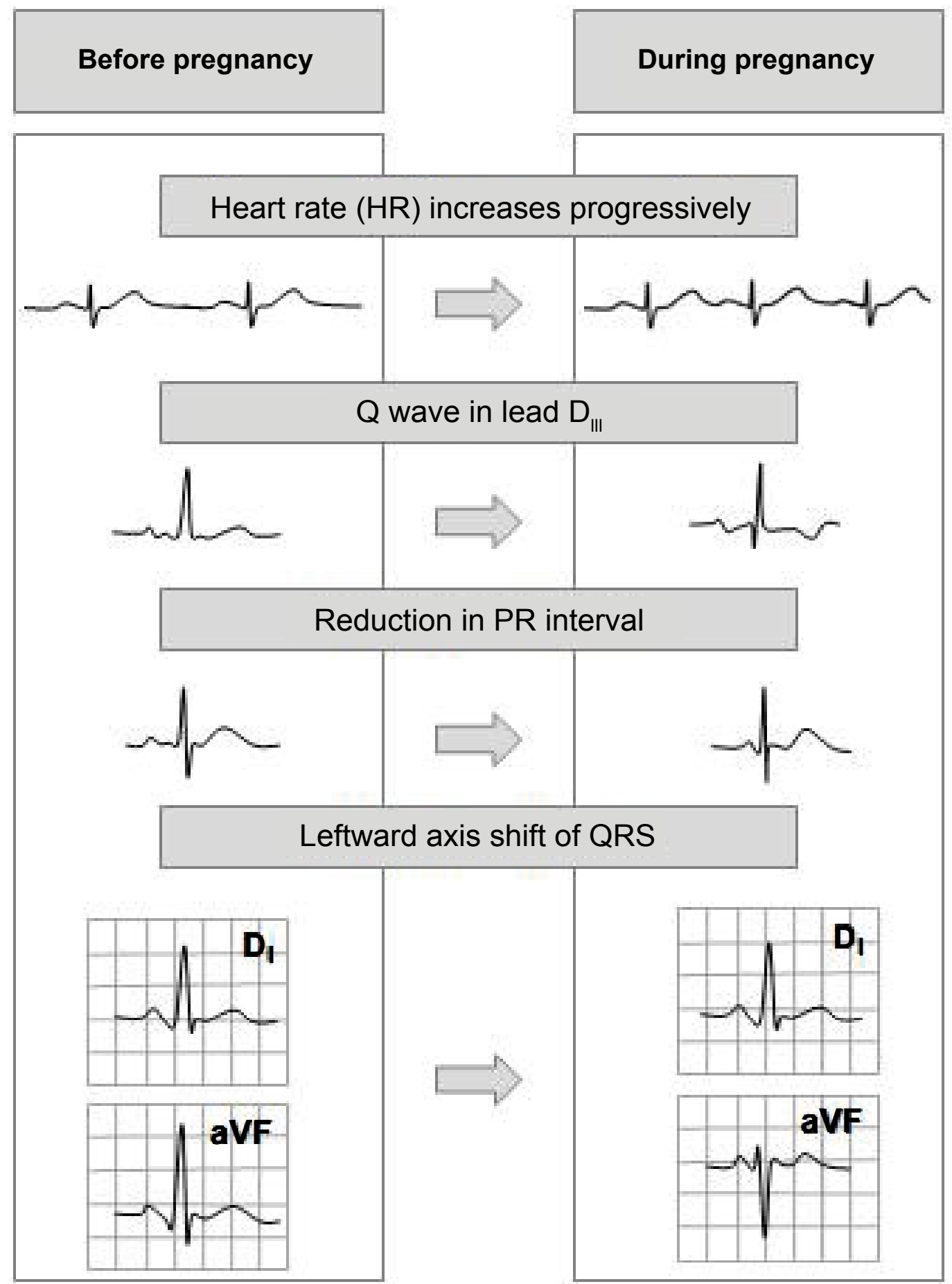

Figure 2: ECG changes in normal pregnancy [48]. Heart rate increases progressively throughout the pregnancy, reaching a peak during the third trimester. Gestational age also impacts QRS complex and T waves, promoting a leftward axis shift as pregnancy progresses. PR interval exhibits a significant reduction in the mean values during pregnancy, while the QRS amplitude generally increases slightly in the late pregnancy.

women enrolled between January and July 2014 at Zekai Tahir Burak Women's Health Training and Research Hospital of Turkey. Specifically, maximum (Pmax) and minimum (Pmin) P-wave durations were defined as the longest and shortest measurable $\mathrm{P}$-wave durations, respectively, in any lead. P-wave dispersion (Pd) was calculated as the maximum minus minimum $P$-wave duration [52]. They demonstrated that the Pd values of the severe preeclampsia group (mean age $28.5 \pm 5.6$ ) were significantly higher compared to that of the mild preeclampsia group (mean age $28.2 \pm 5.5$ ) [52].
These findings are consistent with a recent prospective collaborative screening study between gynecologists, internists and cardiologists $[47,48]$ from Italy, investigating the potential additive role of standard ECG in the identification of Caucasian women at increased risk for hypertensive complications [47].

$P$ wave morphology was analyzed in all of the standard leads. The criteria used for the diagnosis of $\mathrm{P}$ wave abnormality in lead $V_{1}$ were: (1) Bipeak interval in deeply notched $\mathrm{P}$ wave with (2) Terminal forces equal to or more negative than $-0.04 \mathrm{~mm} \cdot \mathrm{sec}$, as obtained from the 
product of the depth of the terminal negative deflection and its duration $[53,54]$. The following other criteria were used for the diagnosis of left atrial abnormality in any other lead than $V_{1}$ : (1) Bipeak interval in deeply notched $P$ waves wider than $0.04 \mathrm{sec}$ or (2) P-wave/ PR-segment ratio greater than 1.6 or (3) P wave higher than $3 \mathrm{~mm}$ or (4) Total $P$ wave duration greater than $0.11 \mathrm{sec}[53,54]$. The primary outcome of the study was the development of gestational hypertension, pre-eclampsia and eclampsia. The secondary outcome was a composite measure of hypertensive disorders and other pregnancy complications including fetal growth restriction, HELLP syndrome, placental abruption, stillbirth, premature delivery and neonatal death $[1,55,56]$. A total of 221 pregnant women were included in the final analysis [47]. Gestational hypertension occurred in 22 women, 5 women experienced pre-eclampsia (3 of these developed HELLP syndrome) and 1 woman had eclampsia. The secondary composite outcome was recorded in 43 women. Multiple events (hypertensive disorders and other maternal or fetal/neonatal complications) were observed in 9 women.

Overall, premature deliveries occurred in 14 women, 6 women delivered growth-restricted neonates, 2 women experienced placental abruption and 2 congenital heart defect requiring admission to neonatal nursery were recorded. At entry, left atrial abnormality in lead $\mathrm{V}_{1}$ was more prevalent in women with hypertension disorders $(p=0.002)$. Age, laboratory tests, HR and other ECG parameters including QT interval and left atrial abnormality observed in other leads than $\mathrm{V}_{1}$ did not differ between the two groups. In a multivariable model, MAP and left atrial abnormality in lead $V_{1}$ were independent predictors of hypertensive disorders. In particular, the presence of left atrial abnormality in lead $\mathrm{V}_{1}$ was associated to a 4-fold increased risk of developing hypertensive disorders (OR: 4.35; 95\% $\mathrm{Cl}: 1.84-10.31 ; \mathrm{p}=0.001$ ).

Of note, the same prediction model also proved significance to identify pregnant women at increased risk for the occurrence of maternal and fetal/neonatal complications [47].

Abnormality of P-wave morphology in lead $\mathrm{V}_{1}$ tested as predictor of hypertensive disorders during pregnancy [47] is commonly used as an ECG sign of left atrial enlargement and it may be easily diagnosed by traditional visual interpretation of ECG tracings, without any need of digitalization or other computer facilities [7].

The mechanisms linking left atrial abnormality on ECG with hypertensive disorders are still elusive [48]. However, several mechanisms, possibly reflected by abnormal left atrial activation on ECG has been suggested. These include increased reactivity to angiotensin II and up-regulation of angiotensin type 1 receptors, with activation of auto-antibodies targeting these receptors [48].

Finally, left ventricular hypertrophy has been suggested to mediate the relation between hypertension and left atrial enlargement [57].

The presence of abnormal $P$ wave morphology at ECG may be a marker of abnormal left ventricular mass which commonly develop in both early and late onset preeclampsia [58-61].

In this context, a recent retrospective study of pregnant women with chronic hypertension [62] showed that women with left ventricular hypertrophy were at greater risk to be delivered preterm $(p=0.001)$, to develop superimposed preeclampsia $(p=0.028)$, and to have an infant requiring intensive care $(p=0.023)$ when compared with those without ventricular hypertrophy. Notably, these findings persisted after adjustment for age, race, and parity [62].

Nevertheless, echocardiographic data on the relationship between the simultaneous adaptations of left ventricle and left atrium during hypertensive disorders of pregnancy are scarce and conflicting [63-65].

\section{Conclusions and Perspectives}

At present, there is considerable interest for prevention of hypertensive disorders of pregnancy, for which our therapeutic approaches are still limited.

Some reports on routine antenatal care suggest that a woman's level of risk for hypertensive disorders may be quantified [7]. In this context, an effective screening for hypertensive disorders might be achieved in the first-trimester of pregnancy using standard ECG in combination with maternal characteristics and history $[7,39,47,48]$.

To better understand potential clinical implications of ECG, we propose an algorithm for specialist referral based on observations from recent studies (Figure 3).

In the first stage, women with family history of hypertensive disorders during pregnancy or with at least one major risk factor for these conditions (preexisting chronic hypertension, chronic vascular and renal disease, connective tissue disease, dyslipidemia, diabetes, obesity, age more than 40 years and multifetal gestation) should be referred to a specialist to perform a standard ECG in the first trimester of pregnancy. The presence of an abnormal $\mathrm{P}$ wave morphology identifies women at high risk for the development of hypertensive disorders during pregnancy. Among those with normal $P$ wave morphology, an uterine Doppler ultrasound with their fetal anatomy scan may be performed to better stratify the risk (specialist referral). Of the women referred for a specialist opinion, ABPM may be also performed during pregnancy to detect masked hypertension and to evaluate pulse pressure and night-time BP.

The primary aim of the proposed new approach to prenatal care is to identify women that would potentially benefit from a closer surveillance and from a prophylactic pharmacological interventions to improve placentation (Figure 3). 


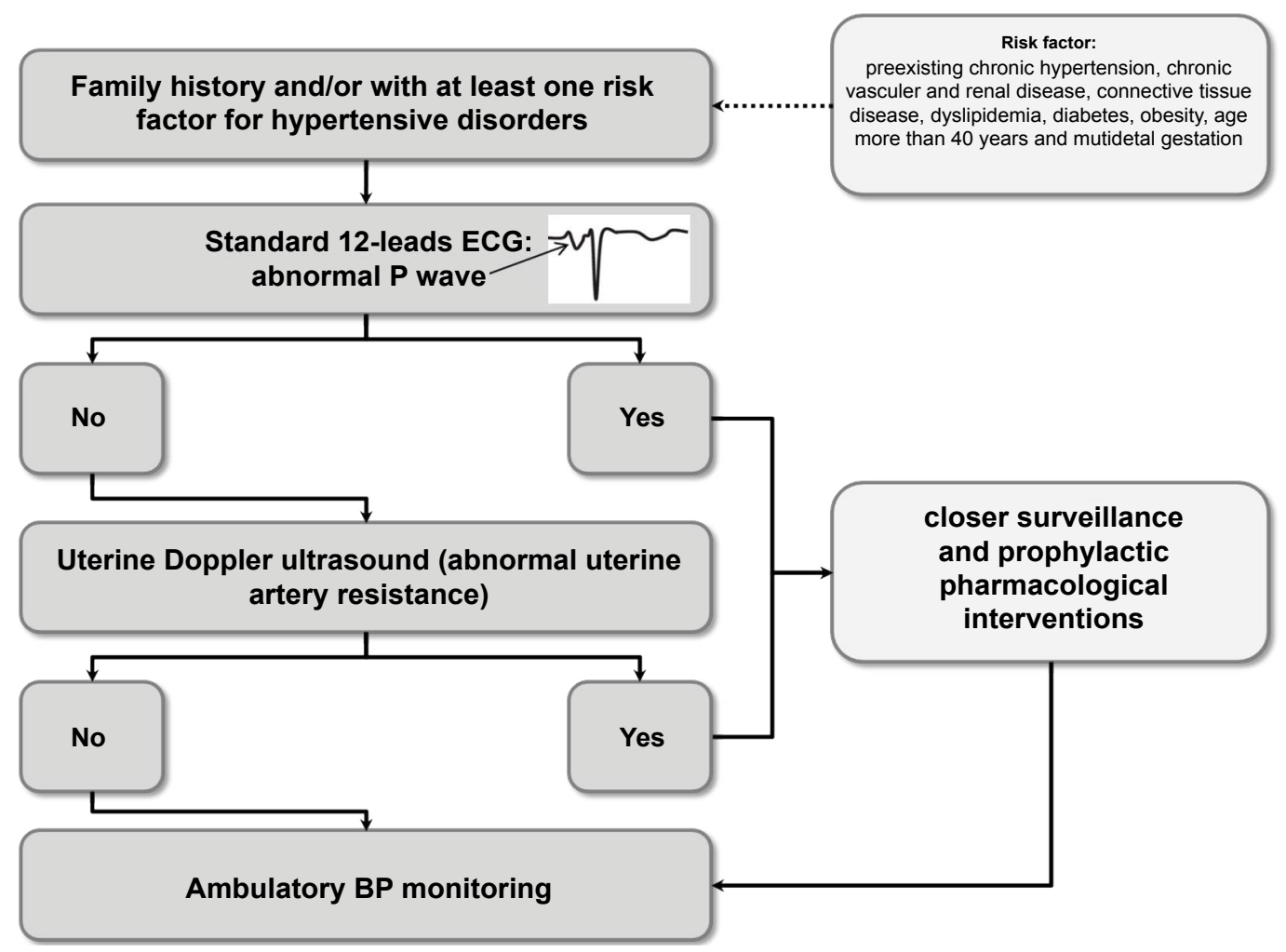

Figure 3: Algorithm for the risk stratification of hypertensive disorders during pregnancy.

Nevertheless, the clinical value of such integrated clinic in which maternal characteristics and history are combined with the results of instrumental techniques to assess the risk for a wide range of hypertensive complications needs to be the subject of well designed prospective studies.

\section{Conflict of Interests}

The authors declare that there is no conflict of interests regarding the publication of this paper.

\section{References}

1. (2000) Report of the National High Blood Pressure Education Program working group on High Blood Pressure in Pregnancy. Am J Obstet Gynecol 183: S1-S22.

2. Magee LA, Pels A, Helewa M, Rey E, von Dadelszen $P$, et al. (2014) Diagnosis, evaluation, and management of the hypertensive disorders of pregnancy: Executive summary. J Obstet Gynaecol Can 36: 416-441.

3. Cunningham FG (2009) Pregnancy hypertension. In: Cunningham FG, Williams Obstetrics. (23 ${ }^{\text {rd }}$ edn), McGraw-Hill Professional, 706.

4. Berg CJ, Chang J, Callaghan WM, Whitehead SJ (2003) Pregnancy-related mortality in the United States, 19911997. Obstet Gynecol 101: 289-296.

5. Chang J, Elam-Evans LD, Berg CJ, Herndon J, Flowers L, et al. (2003) Pregnancy-related mortality surveillance--United States, 1991-1999. MMWR Surveill Summ 52: 1-8.

6. MacKay AP, Berg CJ, Duran C, Chang J, Rosenberg H (2005) An assessment of pregnancy-related mortality in the United States. Paediatr Perinat Epidemiol 19: 206-214.

7. Angeli F, Angeli E, Reboldi G, Verdecchia P (2011) Hypertensive disorders during pregnancy: Clinical applicability of risk prediction models. J Hypertens 29: 2320-2323.

8. Haynes RB, Kastner M, Wilczynski NL (2005) Developing optimal search strategies for detecting clinically sound and relevant causation studies in EMBASE. BMC Med Inform Decis Mak 5: 8.

9. Haynes RB, Wilczynski N, McKibbon KA, Walker CJ, Sinclair JC (1994) Developing optimal search strategies for detecting clinically sound studies in MEDLINE. J Am Med Inform Assoc 1: 447-458.

10. Poon LC, Kametas NA, Valencia C, Chelemen T, Nicolaides KH (2011) Hypertensive disorders in pregnancy: Screening by systolic diastolic and mean arterial pressure at 11-13 weeks. Hypertens Pregnancy 30: 93-107.

11. Poon LC, Kametas NA, Maiz N, Akolekar R, Nicolaides KH (2009) First-trimester prediction of hypertensive disorders in pregnancy. Hypertension 53: 812-818.

12. Audibert F, Benchimol Y, Benattar C, Champagne C, Frydman R (2005) Prediction of preeclampsia or intrauterine growth restriction by second trimester serum screening and uterine Doppler velocimetry. Fetal Diagn Ther 20: 48-53.

13. Dugoff L, Hobbins JC, Malone FD, Porter TF, Luthy D, et al. (2004) First-trimester maternal serum PAPP-A and free-beta subunit human chorionic gonadotropin concentrations and nuchal translucency are associated with obstetric complications: A population-based screening study (the FASTER Trial). Am J Obstet Gynecol 191: 1446-1451.

14. Halligan A, Bonnar J, Sheppard B, Darling M, Walshe J (1994) Haemostatic, fibrinolytic and endothelial variables in normal pregnancies and pre-eclampsia. Br J Obstet Gynaecol 101: 488-492.

15. Hershkovitz R, de Swiet M, Kingdom J (2005) Mid-trimester placentation assessment in high-risk pregnancies using maternal serum screening and uterine artery Doppler. Hypertens Pregnancy 24: 273-280.

16. Kurdi W, Campbell S, Aquilina J, England P, Harrington $\mathrm{K}$ 
(1998) The role of color Doppler imaging of the uterine arteries at 20 weeks' gestation in stratifying antenatal care. Ultrasound Obstet Gynecol 12: 339-345.

17. Milne F, Redman C, Walker J, Baker P, Bradley J, et al. (2005) The pre-eclampsia community guideline (PRECOG): How to screen for and detect onset of pre-eclampsia in the community. BMJ 330: 576-580.

18. Salomon O, Seligsohn U, Steinberg DM, Zalel Y, Lerner A, et al. (2004) The common prothrombotic factors in nulliparous women do not compromise blood flow in the feto-maternal circulation and are not associated with preeclampsia or intrauterine growth restriction. Am J Obstet Gynecol 191: 2002-2009.

19. Steegers-Theunissen RP, Van lersel CA, Peer PG, Nelen WL, Steegers EA (2004) Hyperhomocysteinemia, pregnancy complications, and the timing of investigation. Obstet Gynecol 104: 336-343.

20. Yu CK, Smith GC, Papageorghiou AT, Cacho AM, Nicolaides $\mathrm{KH}$, et al. (2005) An integrated model for the prediction of preeclampsia using maternal factors and uterine artery Doppler velocimetry in unselected low-risk women. Am J Obstet Gynecol 193: 429-436.

21. Briceño-Pérez $C$, Briceño-Sanabria L, Vigil-De Gracia $P$ (2009) Prediction and prevention of preeclampsia. Hypertens Pregnancy 28: 138-155.

22. Cnossen JS, ter Riet G, Mol BW, van der Post JA, Leeflang $\mathrm{MM}$, et al. (2009) Are tests for predicting pre-eclampsia good enough to make screening viable? A review of reviews and critical appraisal. Acta Obstet Gynecol Scand 88: 758-765.

23. Conde-Agudelo A, Villar J, Lindheimer M (2004) World Health Organization systematic review of screening tests for preeclampsia. Obstet Gynecol 104: 1367-1391.

24. Meads CA, Cnossen JS, Meher S, Juarez-Garcia A, ter Riet $\mathrm{G}$, et al. (2008) Methods of prediction and prevention of pre-eclampsia: Systematic reviews of accuracy and effectiveness literature with economic modelling. Health Technol Assess 12: iii-iv, 1-270.

25. Singh HJ (1995) Serum and urinary divalent cations and plasma renin activity in women with mild pregnancy-induced hypertension. Med J Malaysia 50: 93-100.

26. He L, Lang L, Li Y, Liu Q, Yao Y (2016) Comparison of serum zinc, calcium, and magnesium concentrations in women with pregnancy-induced hypertension and healthy pregnant women: A meta-analysis. Hypertens Pregnancy 35: 202-209.

27. Bellomo G, Narducci PL, Rondoni F, Pastorelli G, Stangoni G, et al. (1999) Prognostic value of 24-hour blood pressure in pregnancy. JAMA 282: 1447-1452.

28. Brown MA, Bowyer L, McHugh L, Davis GK, Mangos GJ, et al. (2001) Twenty-four-hour automated blood pressure monitoring as a predictor of preeclampsia. Am J Obstet Gynecol 185: 618-622.

29. Brown MA, Davis GK, McHugh L (2001) The prevalence and clinical significance of nocturnal hypertension in pregnancy. J Hypertens 19: 1437-1444.

30. Hermida RC, Ayala DE (2002) Prognostic value of office and ambulatory blood pressure measurements in pregnancy. Hypertension 40: 298-303.

31. Ozkaya U, Ozkan S, Ozeren S, Corakçi A (2007) Doppler examination of uteroplacental circulation in early pregnancy: Can it predict adverse outcome? J Clin Ultrasound 35 382-386.
32. Scandiuzzi RM, Prado CA, Araujo Júnior E, Duarte G, Quintana SM, et al. (2016) Maternal uterine artery Doppler in the first and second trimesters as screening method for hypertensive disorders and adverse perinatal outcomes in low-risk pregnancies. Obstet Gynecol Sci 59: 347-356.

33. Gómez O, Figueras F, Fernández S, Bennasar M, Martínez $\mathrm{JM}$, et al. (2008) Reference ranges for uterine artery mean pulsatility index at 11-41 weeks of gestation. Ultrasound Obstet Gynecol 32: 128-132.

34. Gómez O, Martínez JM, Figueras F, Del Río M, Borobio V, et al. (2005) Uterine artery Doppler at 11-14 weeks of gestation to screen for hypertensive disorders and associated complications in an unselected population. Ultrasound Obstet Gynecol 26: 490-494.

35. Spencer K, Yu CK, Cowans NJ, Otigbah C, Nicolaides KH (2005) Prediction of pregnancy complications by first-trimester maternal serum PAPP-A and free beta-hCG and with second-trimester uterine artery Doppler. Prenat Diagn 25: 949-953.

36. Spencer K, Yu CKH, Savvidou M, Papageorghiou AT, Nicolaides KH (2006) Prediction of pre-eclampsia by uterine artery Doppler ultrasonography and maternal serum pregnancy-associated plasma protein-A, free beta-human chorionic gonadotropin, activin $A$ and inhibin $A$ at $22+0$ to $24+6$ weeks' gestation. Ultrasound Obstet Gynecol 27: 658-663.

37. Cnossen JS, Morris RK, ter Riet G, Mol BW, van der Post JA, et al. (2008) Use of uterine artery Doppler ultrasonography to predict pre-eclampsia and intrauterine growth restriction: A systematic review and bivariable meta-analysis. CMAJ 178: 701-711.

38. Carruth JE, Mivis SB, Brogan DR, Wenger NK (1981) The electrocardiogram in normal pregnancy. Am Heart $\mathrm{J} 102$ : 1075-1078.

39. Angeli F, Angeli E, Verdecchia P (2014) Electrocardiographic changes in hypertensive disorders of pregnancy. Hypertens Res 37: 973-975.

40. Akinwusi PO, Oboro VO, Adebayo RA, Akintunde AA, Adeniji AO, et al. (2011) Cardiovascular and electrocardiographic changes in Nigerians with a normal pregnancy. Cardiovasc J Afr 22: 71-75.

41. Boyle DM, Lloyd-Jones RL (1966) The electrocardiographic ST segment in pregnancy. J Obstet Gynaecol Br Commonw 73: 986-987.

42. Grand A, Rigaud-Morel N, Drouin B, Boissel J, Burel H (1983) Electrocardiographic changes induced by pregnancy in healthy women. Arch Mal Coeur Vaiss 76: 77-86.

43. Gregorini L, Valentini R, Lo Cicero G, Ferrari A, Mancia G (1976) Electrocardiographic and vectorcardiographic modifications during pregnancy and post partum. Boll Soc Ital Cardiol 21: 2049-2054.

44. Halphen C, Leguludec D, Valent R, Haïat R (1984) Electrocardiographic study of left ventricular performance in normal pregnancy. Arch Mal Coeur Vaiss 77: 212-217.

45. Illanes A, Droguett A, Fuentealba A (1953) Electrocardiographic studies on normal pregnancy. Rev Med Chil 81: 152-159.

46. Wenger NK, Hurst JW, Strozier VN (1964) Electrocardiographic changes in pregnancy. Am J Cardiol 13: 774-778.

47. Angeli E, Verdecchia P, Narducci P, Angeli F (2011) Additive value of standard ECG for the risk prediction of hypertensive disorders during pregnancy. Hypertens Res 34: 707-713. 
48. Angeli F, Angeli E, Verdecchia P (2015) Novel Electrocardiographic patterns for the prediction of hypertensive disorders of pregnancy-from pathophysiology to practical implications. Int J Mol Sci 16: 18454-18473.

49. Isezuo SA, Ekele BA (2004) Eclampsia and abnormal QTc. West Afr J Med 23: 123-127.

50. Baumert M, Seeck A, Faber R, Nalivaiko E, Voss A (2010) Longitudinal changes in $Q T$ interval variability and rate adaptation in pregnancies with normal and abnormal uterine perfusion. Hypertens Res 33: 555-560.

51. Raffaelli R, Prioli MA, Parissone F, Prati D, Carli M, et al. (2014) Pre-eclampsia: Evidence of altered ventricular repolarization by standard ECG parameters and QT dispersion. Hypertens Res 37: 984-988.

52. Kirbas O, Biberoglu EH, Kirbas A, Daglar HK, Kurmus O, et al. (2014) P-wave duration changes and dispersion in preeclampsia. Eur J Obstet Gynecol Reprod Biol 183: 141145.

53. Tarazi RC, Miller A, Frohlich ED, Dustan HP (1966) Electrocardiographic changes reflecting left atrial abnormality in hypertension. Circulation 34: 818-822.

54. Munuswamy K, Alpert MA, Martin RH, Whiting RB, Mechlin NJ (1984) Sensitivity and specificity of commonly used electrocardiographic criteria for left atrial enlargement determined by M-mode echocardiography. Am J Cardiol 53: 829-832.

55. Lenfant C, National Education Program Working Group on High Blood Pressure in Pregnancy (2001) Working group report on high blood pressure in pregnancy. J Clin Hypertens (Greenwich) 3: 75-88.

56. Lindheimer MD, Taler SJ, Cunningham FG (2008) Hypertension in pregnancy. J Am Soc Hypertens 4: 68-78.

57. Gerdts E, Oikarinen L, Palmieri V, Otterstad JE, Wachtell $\mathrm{K}$, et al. (2002) Correlates of left atrial size in hypertensive patients with left ventricular hypertrophy: The Losartan Intervention For Endpoint Reduction in Hypertension (LIFE) Study. Hypertension 39: 739-743.

58. Novelli GP, Valensise H, Vasapollo B, Larciprete G, Altomare $F$, et al. (2003) Left ventricular concentric geometry as a risk factor in gestational hypertension. Hypertension 41: 469-475.

59. Valensise H, Vasapollo $B$, Novelli GP, Pasqualetti $P$, Galante A, et al. (2006) Maternal total vascular resistance and concentric geometry: A key to identify uncomplicated gestational hypertension. BJOG 113: 1044-1052.

60. Vasapollo B, Novelli GP, Valensise H (2008) Total vascular resistance and left ventricular morphology as screening tools for complications in pregnancy. Hypertension 51: 1020-1026.

61. Valensise H, Vasapollo B, Gagliardi G, Novelli GP (2008) Early and late preeclampsia: Two different maternal hemodynamic states in the latent phase of the disease. Hypertension 52: 873-880.

62. Ambia AM, Morgan JL, Wilson KL, Roberts SW, Wells CE, et al. (2017) Frequency and consequences of ventricular hypertrophy in pregnant women with treated chronic hypertension. Am J Obstet Gynecol 217: 467.e1-467.e6.

63. Ingec M, Yilmaz M, Gundogdu F (2005) Left atrial mechanical functions in pre-eclampsia. J Obstet Gynaecol Res 31: 535-539.

64. Borghi C, Esposti DD, Immordino V, Cassani A, Boschi S, et al. (2000) Relationship of systemic hemodynamics, left ventricular structure and function, and plasma natriuretic peptide concentrations during pregnancy complicated by preeclampsia. Am J Obstet Gynecol 183: 140-147.

65. Vázquez Blanco M, Grosso O, Bellido CA, lavícoli OR, Berensztein CS, et al. (2001) Dimensions of the left ventricle, atrium, and aortic root in pregnancy-induced hypertension. Am J Hypertens 14: 390-392. 